\title{
Regulating the dynamic folding of a DNA hairpin at the expense of a small, molecular fuel
}

\author{
Michele Stasi, Alba Monferrer, Leon Babl, Sreekar Wunnava, Christina Dirscherl, Dieter Braun, Petra Schwille, \\ Hendrik Dietz, Job Boekhoven
}

KEYWORDS: DNA Nanotechnology, Chemical Fuel, Non-equilibrium, DNA Machines

\begin{abstract}
Molecular machines, such as ATPases or motor proteins, couple the catalysis of a chemical reaction, most commonly hydrolysis of nucleotide triphosphates, to their conformational change. In essence, they continuously convert a chemical fuel to drive their motion. An outstanding goal of nanotechnology remains to synthesize a nanomachine with similar functions, precision, and speed. The field of DNA nanotechnology has given rise to the engineering precision required for such a device. Simultaneously, the field of systems chemistry developed fast chemical reaction cycles that convert fuel to change the function of molecules. In this work, we thus combined a fast, chemical reaction cycle with the precision of DNA nanotechnology to yield kinetic control over the conformational state of a DNA hairpin. Future work on such systems will result in fast and precise DNA nanodevices.
\end{abstract}

\section{INTRODUCTION}

Nucleic acids have proven to be outstanding building blocks for molecular self-assembly. The precision and fidelity of base-pairing allows for predicting self-assembly patterns and optimization of the strength of the interaction. The advent of DNA origami ${ }^{1-4}$ has pushed further the boundaries of the possible structures designed with DNA, opening the door for the easy bottom-up assembly of user-defined three-dimensional structures with unprecedented accuracy. Although initial works in the field have mostly focused developing static structures, so-called structural DNA nanotechnology, a strong interest is now diverted towards the development of dynamic systems that reshape and actively interact with their surroundings ${ }^{5-8}$.

If structural DNA nanotechnology enables to build virtually any architecture on the nanometer scale, the combination with actuation mechanisms could fulfill the dream of a fully synthetic biomolecular machine ${ }^{7}$. Several strategies to control the actuation of DNA nanodevices have been employed. Toehold-mediated strand displacement reaction ${ }^{9-15}$ is a popular approach that relies on user-designed DNA sequences with partial complementarity making it easy to implement. A limiting factor in this strategy is the $2^{\text {nd }}$ order kinetic of hybridization between DNA sequences that require high concentrations of DNA strands to achieve operational speed in the range of seconds. Alternative approaches for actuation of DNA-based nanodevices include light-responsive molecules ${ }^{16}$, redox stim$\mathrm{uli}^{17,18}, \mathrm{pH}$-dependent secondary structures ${ }^{19-21}$, actuation by variating the ionic strength of the medium ${ }^{22}$ or the temperature and thus controlling the stacking interaction of nucleobases in blunt ends, through the use of external electric or magnetic fields ${ }^{23,24}$, or biomolecules such as antibodies ${ }^{25}$ and enzymes ${ }^{26-29}$. Most of these strategies proceed stepwise, i.e., the structure switches from one static state to another upon application of the stimulus. Additionally, they operate profoundly differently compared to naturally occurring biomolecular machines in the way they harness the energy required to function. Specifically, the dynamics in synthetic examples either result from a change in the overall environment (e.g., by changing $\mathrm{pH}$, ionic strength), thereby irreversibly driving the system into a new global minimum, or, in the case of light, lifts the system to a different energy landscape. Conversely, natural molecular machines, such as ATPases or motor proteins, couple the catalysis of a chemical reaction, e.g., the hydrolysis of nucleotide triphosphates, to their conformational change: they incessantly consume fuel to drive their motion ${ }^{30}$.

The constant conversion of fuels to drive the function of molecules has also been applied to the self-assembly of small molecules. ${ }^{31}$,

${ }^{32}$ In such a chemically fueled system, molecular assembly is regulated by a fuel-driven reaction cycle that comprises at least two reactions, i.e., an activation and deactivation reaction. In the activation reaction, a precursor reacts with a chemical fuel which activates it for self-assembly. In the deactivation, the activated product spontaneously reverts to the precursor. In its finite lifetime, the activated product can be temporarily part of a dynamic assembly which is thus regulated by the kinetics of the reaction cycle. This strategy has resulted chemically fueled dynamic fibers ${ }^{33,34}$, droplets ${ }^{35}$, colloids ${ }^{36}$, and small molecular machines ${ }^{37}$. Developments in chemically fueled self-assembly have resulted in fast reaction cycles in which activated products have half-lives in the range of tens of seconds. Nevertheless, the field of chemically fueled self-assembly of small molecules still lacks precision and designability when compared to DNA nanotechnology.

Therefore, we combined a fast reaction cycle with the precision of DNA nanotechnology to achieve kinetic control over the conformational state of a DNA hairpin (Scheme 1). The system can spontaneously revert to its initial state once the fuel is fully consumed. The molecular design we present is versatile, can be easily introduced in commercially available DNA sequences, and provides a strategy towards non-equilibrium self-assembly and actuation in DNA nanotechnology. 


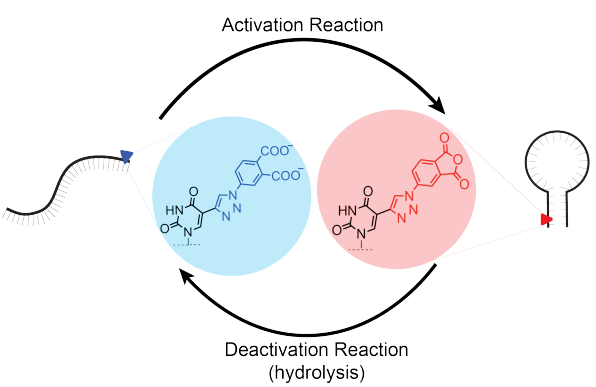

Scheme 1. Schematic representation of the fuel-driven hairpin. The hairpin is mostly unfolded state in its precursor state (dicarboxylic acid, blue). Upon activation (anhydride, red), the folded state becomes more stable.

\section{RESULTS AND DISCUSSION}

We used a versatile, well-described chemical reaction cycle ${ }^{38-40}$ in which a phthalic acid-based precursor is transiently activated into its corresponding anhydride at the expense of a molecule of EDC (1ethyl-3-(3-dimethylaminopropyl)carbodiimide, Fig. 1A). In the activation reaction, the precursor reacts with EDC to yield the precursor's anhydride state and EDU (1-(3-(dimethylamino)propyl)-3ethylurea) in a two-step process. The anhydride product rapidly hydrolyzes to its precursor state in the aqueous environment, i.e., the deactivation reaction. In its finite lifetime, the hydrophobization and rigidification of the molecule can induce self-assembly or, in our design, affect the stability of a DNA hairpin. Using copper-catalyzed alkyne-azide cycloaddition reaction, we modified an alkyne-bearing thymine to carry two carboxylates that serve as the precursor for our reaction cycle (modified thymine, Fig. 1A).
First, we tested the kinetics of the nucleotide itself, i.e., without incorporating it in a DNA hairpin (modified thymine, Fig. 1A). The addition of $4 \mathrm{mM}$ EDC to $8 \mathrm{mM}$ of the modified thymine in $200 \mathrm{mM}$ MES at $\mathrm{pH} 6$ yielded the formation of the corresponding anhydride product, as evidenced by HPLC. As the reaction progressed, both the concentrations of fuel and anhydride decayed (Fig. S1). The kinetics of the reaction cycle could be accurately captured with a kinetic model (See Supporting Note 1), which allowed us to calculate the half-life of the anhydride product $\left(t_{1 / 2}\right)$ to be $23 \mathrm{sec}$.

Next, we incorporated the modified thymine into a molecular beacon comprising a $\mathrm{Cy} 3$ dye on the 5 ' end and a black hole quencher (BHQ-2) on the 3 ' end of a 30-base oligomer (Fig. 1B) and measured its melting temperature by fluorescence spectroscopy (see Fig S2). The molecular beacon we chose is based on a hairpin that, without any artificial modification, has a melting temperature $\left(\mathrm{T}_{\mathrm{m}}\right)$ of $40.4^{\circ} \mathrm{C}$ under the applied conditions (H-0, Fig. 1C). We anticipated that incorporating one modified thymine would decrease the $\mathrm{T}_{\mathrm{m}}$ due to steric hindrance and electrostatic repulsion of the modified thymine compared to regular thymine. Thus, we designed $\mathrm{H}-1$, which is $\mathrm{H}-0$ with a point-mutation of one thymine for modified thymine in the stem region of the molecular beacon. As expected, we found that its melting temperature was $10.5^{\circ} \mathrm{C}$ lower compared to $\mathrm{H}-0$. This decrease in $\mathrm{T}_{\mathrm{m}}$ was independent of where the mutation was performed (H-2), provided it was in the stem region, i.e., $\mathrm{H}-3$ was modified in the loop region, and its $\mathrm{T}_{\mathrm{m}}$ was almost equal to the unmodified $\mathrm{H}-0$. If we added two mutations in the stem region, $T_{m}$ decreased by 20.6 ${ }^{\circ} \mathrm{C}$ (H-4). Taken together, the incorporation of modified thymines in the stem region destabilizes the duplex.
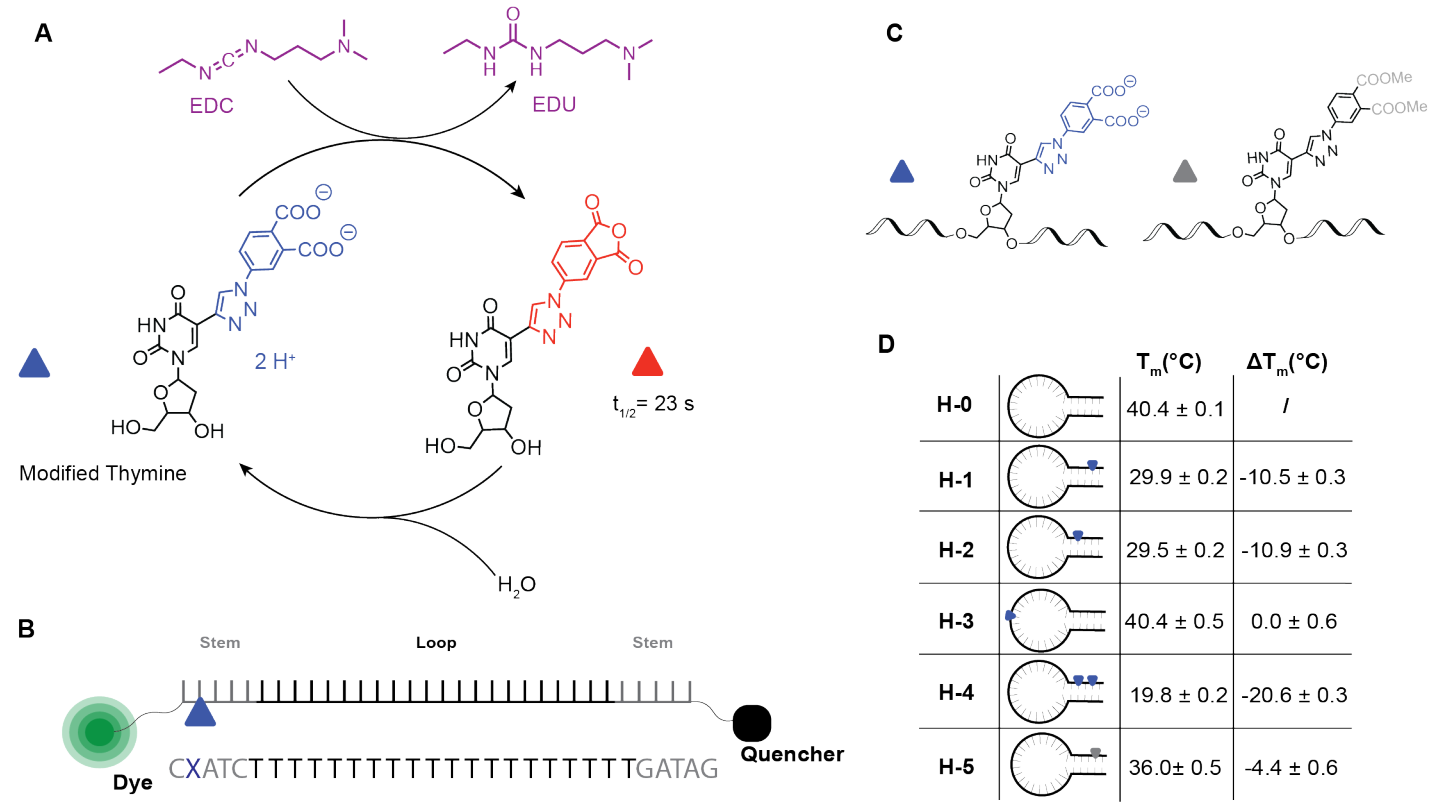

Figure 1. The molecular design of the dynamically folding hairpins. (A) The chemical reaction cycle that activates a modified thymine at the expense of a carbodiimide-based fuel. (B) The sequence of the oligomer in which the modified thymine is incorporated. The dye is Cy3, the quencher is a Black Hole Quencher 2, and the blue triangle represents the modified thymine. (C) A schematic representation of an oligomer with the modified thymine incorporated and an oligomer with a control compound, i.e., the methyl ester of the modified thymine, which is represented as a grey triangle. (D) A table of oligomers ( $\mathrm{H}-0$ to $\mathrm{H}-5$ ) with the location of their modification (blue triangles), their melting temperature ( $\left.\mathrm{T}_{\mathrm{m}}\right)$, and the difference in $\mathrm{T}_{\mathrm{m}}$ compared to non-functionalized $\mathrm{H}-0$ as measured by fluorescence melting experiments (See Fig S2).

The destabilizing effect of the modified thymine can be attributed to electrostatic repulsion of the carboxylates and /or steric hindrance of the phthalic acid and triazole in the crowded environment of the duplex. To test which of these forces was the dominant one, we 
synthesized the methyl-ester of modified thymine which lacks the anionic nature compared to modified thymine but is similar in size (Fig. 1C). When incorporated in the molecular beacon $(\mathrm{H}-5)$, we found a small drop in $\mathrm{T}_{\mathrm{m}}\left(4.4^{\circ} \mathrm{C}\right)$ compared to non-modified $\mathrm{H}-0$. This observation suggests that both a steric hindrance and the presence of the negative charges are responsible for the change in the $T_{m}$ upon incorporating modified thymine.

Next, we tested the ability of the chemical reaction cycle to induce the folding of the hairpin. We added $20 \mathrm{mM}$ EDC to $0.2 \mu \mathrm{M} \mathrm{H}-1$ (i.e., a 100.000-fold excess) and monitored the fluorescence intensity of the molecular beacon as a function of time (Fig. 2A). The experiment was performed at the $\mathrm{T}_{\mathrm{m}}$ of $\mathrm{H}-1\left(30^{\circ} \mathrm{C}\right)$, and we can thus assume that roughly $50 \%$ of the hairpin was in its folded state before we initiated the experiment. Immediately after the addition of the EDC, the fluorescence intensity dropped drastically, indicating that the population of folded hairpins had increased. Ten minutes after the EDC addition, we added $10 \mathrm{mMN}$-acetyl aspartate (Ac-D-OH) as a scavenger, i.e., a competing dicarboxylate that can also cyclically react with EDC and rapidly consumes all fuel. Consequently, the hairpin fluorescence returned to a value close to the original level. We attribute the lack of a complete return due to the change in ionic strength of solution caused by the positively charged group present in both EDC and its byproduct of hydration, i.e., the waste EDU (Fig. 2C).

These results suggest that the addition of chemical fuel creates a steady state of anhydride, which changes the dynamic population of the folded hairpins and unfolded oligomers. We quantified the change in the folding caused by the fuel by calculating the amount of folded hairpins as a fraction of the total concentration of oligomers $(\alpha)$ :

$$
\alpha=\frac{[\text { folded hairpins }]}{[\text { oligomers }]}
$$

We determined the concentration of folded hairpins by fluorescence spectroscopy. To do so, we compared the fluorescence intensity of our sample with fuel at $30^{\circ} \mathrm{C}$ to two reference points, i.e., the fluorescence intensity of a sample where all the hairpins were folded and fluorescence intensity at $30^{\circ} \mathrm{C}$ before the addition of fuel. We prepared the sample where all the hairpins were folded by the addition of $20 \mathrm{mM} \mathrm{MgCl} 2$ while the corresponding $\alpha$ at $30^{\circ} \mathrm{C}$ is known from the melting curve of each $\mathrm{H}$ (Supporting Table 1). From these two reference points, the $\alpha$ was calculated using the equation described in Supporting Notes 2.

We measured the increase in $\alpha$ upon the addition of fuel for all the hairpins in this work (Fig. 2C). We compared those values to the increase in $\alpha$ caused by the addition of EDU. As expected, for nonmodified $\mathrm{H}-0$ the increase in $\alpha$ was minimal. Moreover, it was similar to the increase upon the addition of EDU. The same trend is observed for $\mathrm{H}-3$, i.e., the molecular beacon that has the modified thymine in the loop region. For the final control compound $\mathrm{H}-5$, where the carboxylic acids are protected as methyl esters, the change induced by the fuel and the waste was modest, and we attribute it to the change in the ionic strength of the solution. In contrast, $\mathrm{H}-1, \mathrm{H}-2$, and $\mathrm{H}-4$ displayed a striking difference between the addition of EDC or EDU. In the case of $\mathrm{H}-4$, we started with an almost open state before applying fuel $(\alpha=0.21)$. The addition of fuel pushed the system towards a closed state $(\alpha=0.66)$. In other words, the fuel switched the system from a condition where the open state is dominant to a new, temporary distribution where the closed state is in excess. We then explored the effect of different amounts of fuel on the folding of $\mathrm{H}-1$. We chose to focus on $\mathrm{H}-1$ because it has only one modified thymine, thus reducing the number of transient species. When increasing amounts of fuel are added, a greater degree of folding is achieved (Fig. 2D), which is mainly attributed to the higher yield of anhydride in a steady state. In contrast, when a similar amount of EDU was added, the change in $\alpha$ was much lower (Fig S3). These combined experiments conclude that the addition of fuel increases the population of folded hairpins by activating the modified thymine.
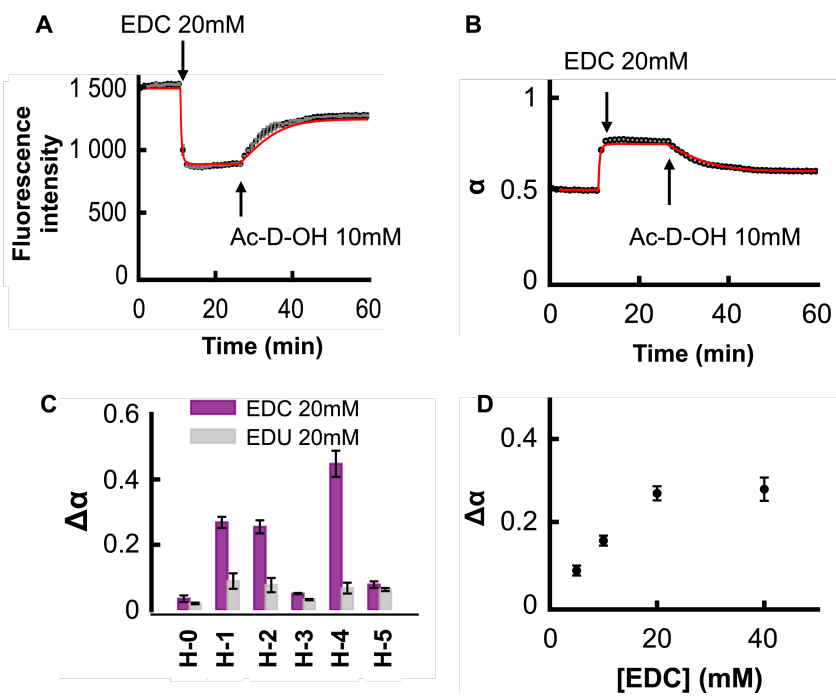

Figure 2. The response of the molecular beacon to chemical fuel. (A) The fluorescence intensity as a function of time for $\mathrm{H}-1$. At $10 \mathrm{~min}$, $20 \mathrm{mM}$ EDC is added. After an additional $15 \mathrm{~min}, 10 \mathrm{mM} \mathrm{N}$-acetyl aspartic acid is added to consume the remaining EDC. Black markers represent the experimental data. The standard deviation of duplicate experiments is shown as error bar. The red line represents a prediction with our kinetic model. (B) The evolution of $\alpha$ for the experiment described in A. (C) Comparison of the effect of fuel (EDC, $20 \mathrm{mM}$ ) and waste (EDU, $20 \mathrm{mM}$ ) on the folding of the different oligonucleotides. (D) Effect of different amounts of fuel on the folding of $\mathrm{H}-1$.

The fluorescence data provided information about the overall fraction of the folded hairpin. However, both precursor and activated states are simultaneously present, and each can form hairpins (See Scheme 2). Our system was designed such that the activated state is more likely to form a hairpin, and we were interested in understanding the contributions of each of the two possible hairpin species on the overall fraction of folded hairpins.

We can rewrite eq. 1 as the sum of two terms like in the following:

$$
\begin{aligned}
\alpha & \left.=\frac{[\text { folded hairpins }]}{[\text { oligomers }]}=\frac{\left[\text { anhydride }_{\text {folded }}\right]+\left[\text { acid }_{\text {folded }}\right]}{[\text { oligomer }]}\right] \\
& =\frac{\alpha_{1} \cdot[\text { oligomers }] \cdot y+\alpha_{0} \cdot[\text { oligomer }] \cdot(1-y)}{[\text { oligomers }]} \\
& =\alpha_{1} \cdot y+\alpha_{0} \cdot(1-y)
\end{aligned}
$$

Where $\alpha_{0}$ and $\alpha_{1}$ are the folding ratio of the acid and the anhydride state, respectively, and $y$ is the steady-state yield of the reaction cycle. To determine those two $\alpha$ values, we thus have to determine the steady-state concentration of anhydride which we measured by HPLC. As a precursor, we used a short oligonucleotide equipped with our modified thymine and we applied different amounts of fuel to induce different steady-state levels. We quenched these samples 
after 1 min with a previously established quenching method ${ }^{41}$. In brief, we added EDC to a solution of unlabeled oligo $(1 \mu \mathrm{M})$ in MES buffer at $\mathrm{pH} 5.5$ and after $1 \mathrm{~min}, 360 \mathrm{mM}$ benzylamine was added to the sample. We found that two new peaks appeared in the chromatogram, which we attributed to the products of the reaction of the anhydride with benzylamine (see Fig. S15-16). By comparing the areas of the peaks, we calculated the yield of anhydride in a steady-state which we used in our kinetic model to fit the fluorescence data as a function of time (See Supporting Note 1 and Fig. S4). From the model, we derived a value for $\alpha_{1}$, which we estimated to be 0.95 . In other words, $95 \%$ of the activated oligos are in their folded state, whereas only $50 \%$ of the nonactivated oligos are in their folded state.

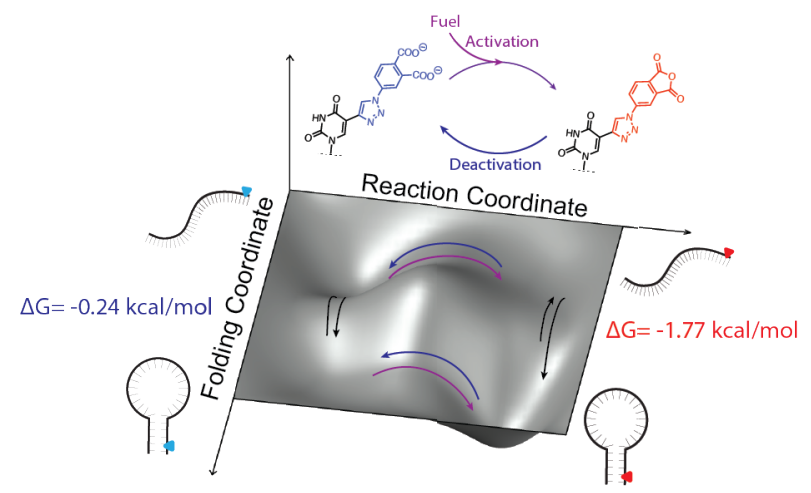

Scheme 2. The proposed energy landscape of the chemically fueled hairpin. A hairpin in our system can toggle between four positions in our energy landscape: unactivated and unfolded, unactivated and folded, activated and unfolded, and activated and unfolded. Chemical activation makes folding more favorable.

The obtained data creates a clearer picture of the energy landscape of our dynamic hairpins (Scheme 2). In the energy landscapes, four states exist: a DNA strand can either be folded or unfolded, and it can either be in an activated or deactivated state. Pathways exist between each of these states, however, only the eight dominant ones are shown. These are: an unactivated strand can fold and unfold, and an activated strand can fold and unfold (pathways along the folding coordinate). Along the reaction coordinate, a folded or unfolded strand can become activated, and a folded and unfolded activated strand can become deactivated. While it is technically possible that a folded oligomer simultaneously deactivates and unfolds, it is unrealistic and complicates the landscape.

We found that the activated state has a half-life of 23 seconds (vide supra). We also found that its degree of folding is in the range of $95 \%$. Thus, in its lifetime, the activated oligomer will spend roughly $95 \%$ of its time in the folded state. However, the half-life for the folding of a DNA strand such as the one used in our experiments is reported to be in the range of milliseconds. ${ }^{42-44}$ That means that even though an activated state will spend roughly $95 \%$ of its time in a folded state, it will unfold and refold 100s of times in its lifetime.

Interestingly, the anhydride state was significantly more folded compared to the methyl ester control compound (activated $\mathrm{H}-1$ vs $\mathrm{H}-5$, $\alpha=0.95$ vs $\alpha=0.71$, under the same conditions) even though both species lack the negative charges. A possible explanation is that the formation of the anhydride increases the overall planarity of the functional group thus facilitating intercalation and stacking into the DNA helix. We also observed a modest increase in the activation rate constant ( $k_{1}$ in the kinetic model, see Supporting Table 2) for the modified thymine when embedded in the oligo, in agreement with the previous report from our group that a densely charged local environment can affect the reactivity of dicarboxylic acid. ${ }^{34}$

In the experiments above, we changed the fraction of folding of hairpins in a steady-state by the addition of fuel. The addition of a fuel-scavenger allows to reversibly control the steady-state by rapidly depleting all fuel. Next, we introduce the idea of a permanently present fuel-scavenger that consumes most fuel and thus controls the lifetime of the folded population. Such an approach opens the door to self-regulating DNA nanodevices with time-dependent behavior. When fuel is added to such a competition experiment, the majority of the fuel is consumed by the scavenger, and the conformation change of the hairpin is only temporary (Fig. 3A). A simulation by our kinetic model demonstrated the validity of this approach and showed that lifetimes of the folded population could be tuned from few minutes to hours depending on the amount of scavenger that is added (Fig. 3B). We carried out these experiments using $0.2 \mu \mathrm{M}$ of $\mathrm{H}-1$ in MES buffer containing variable concentrations of Ac-D-OH as a scavenger at $30^{\circ} \mathrm{C}$. When $20 \mathrm{mM}$ EDC is added to the system with $20 \mathrm{mM}$ of scavenger, we observed a marked drop in the fluorescence, followed by a rapid recovery of the signal (Fig. 3C). The kinetic profile of the fluorescence recovery correlates with the experimentally determined consumption of the EDC (Fig. S6). Moreover, the fluorescence signal is accurately modeled by our kinetic model and correlates with the predicted anhydride concentration (Fig. S7$8)$. The concentration of scavenger could be used to tune the half-life of recovery of the folded states from $4 \mathrm{~min}$ to over $13 \mathrm{~min}$ in agreement with the predicted half-life of recovery (Fig. 3D). The ability of the system to autonomously stop allows operating multiple cycles with minimal intervention, which we demonstrate by the addition of five batches of $20 \mathrm{mM}$ of EDC, which resulted in a transient change in the population of folded and unfolded molecules each time (fig. $3 \mathrm{E})$. In contrast, most of the DNA dynamic system reported so far requires either extensive manipulation, such as buffer exchange, or at least two different stimuli to move between the different states. 
A

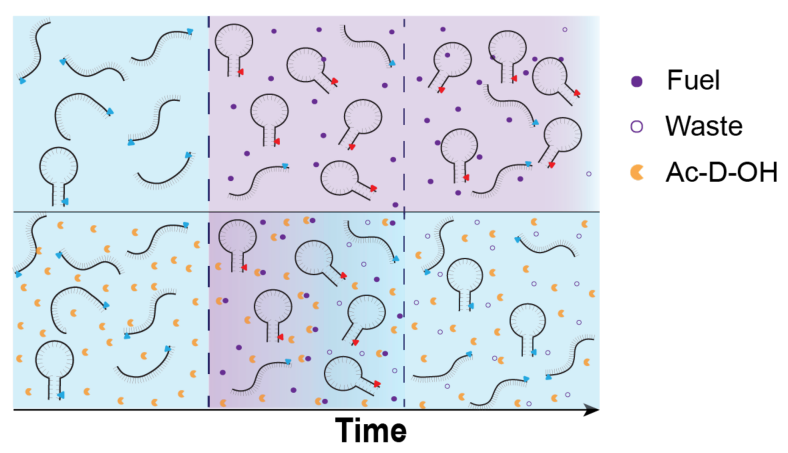

C

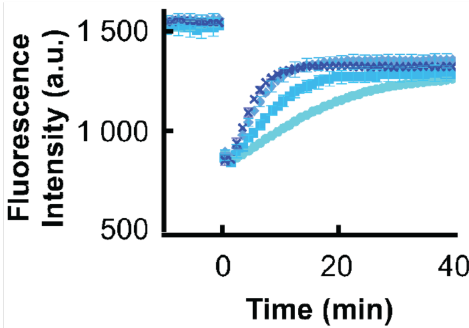

D

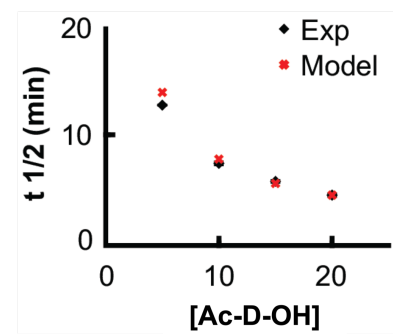

B

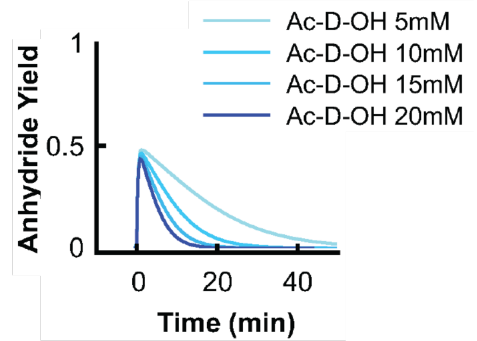

E

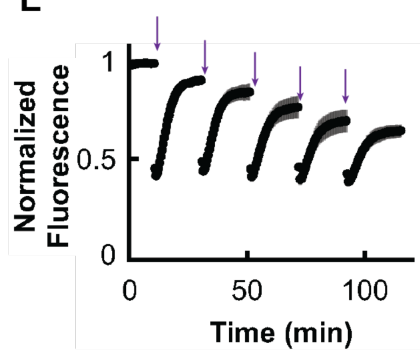

Figure 3. Regulating the lifetime of the folded hairpin population by introducing a fuel-scavenger. (A) Schematic representation of the effect of scavenger (Ac-D-OH) on the system (B) A simulation of the anhydride yield as a function of time in the presence of different amounts of scavenger. (C) The fluorescence intensity of $\mathrm{H}-1$ ( $0.2 \mu \mathrm{M}$ fueled with $20 \mathrm{mM}$ EDC) as a function of time in the presence of different amounts of Ac-D-OH (5, 10, 15, $20 \mathrm{mM}$, respectively). (D) The half-life of fluorescence recovery for experiment in B. (E) Sequential addition of EDC $20 \mathrm{mM}$ to $\mathrm{H}-4(0.2 \mu \mathrm{M})$. Each purple arrow indicates one $20 \mathrm{mM}$ batch of fuel addition.

\section{CONCLUSION}

In conclusion, we used a fuel-driven reaction cycle to alter the likelihood of folding of a DNA hairpin. The reaction cycle operates directly on the DNA sequence, i.e., it acts on a modified thymine bearing two negatively charged carboxylic acids that become neutral and more rigid, which decreases its steric hindrance, electrostatic repulsion, and increases its ability to base stack upon folding. Future single-molecule studies should further elucidate the kinetics of folding and unfolding of this system, giving a complete picture of the energy landscape. Further changes in the molecular design will increase the energy difference between the active and precursor state even further.

In general, this approach is conceptually more similar to the operational mode of biomolecular machines in the sense that the energy of a chemical fuel - an abiotic one in our case - is used to drive a conformational change of the device in a non-equilibrium fashion. We demonstrated that this is a successful strategy to control the hybridization of DNA in a simple model system, and we envision that its implementation in more complex supramolecular structures, such as DNA origami, will open new possibilities to power such structures.

\section{ASSOCIATED CONTENT}

\section{Supporting Information}

The Supporting Information is available free of charge on the ACS Publications website. Materials and methods description, and additional fluorescence data, and HPLC-data (PDF).

\section{AUTHOR INFORMATION}

\section{Corresponding Author}

Job Boekhoven - Department of Chemistry, Technical University of Munich, 85748 Garching, Germany; orcid.org/0000-0002-9126-2430; Email: job.boekhoven@tum.de

\section{Authors}

Michele Stasi - Department of Chemistry, Technical University of Munich, Lichtenbergstrasse 4, 85748 Garching, Germany.

Alba Monferrer - Department of Physics, Technical University of Munich, Am Coulombwall 4, 85748 Garching, Germany

Leon Babl - Max Planck Institute of Biochemistry, Am Klopferspitz 18, 82152 Martinsried, Germany

Sreekar Wunnava - Center for NanoScience (CeNS) and Systems Biophysics Ludwig-Maximilian University Munich, Munich, Germany

Christina Dirscherl - Center for NanoScience (CeNS) and Systems Biophysics Ludwig-Maximilian University Munich, $\mathrm{Mu}-$ nich, Germany

Dieter Braun - Center for NanoScience (CeNS) and Systems Biophysics Ludwig-Maximilian University Munich, Munich, Germany

Petra Schwille - Max Planck Institute of Biochemistry, Am Klopferspitz 18, 82152 Martinsried, Germany

\section{Author Contributions}

The manuscript was written through contributions of all authors. All authors have given approval to the final version of the manuscript. t These authors contributed equally. 


\section{Notes}

The authors declare no competing financial interest.

\section{ACKNOWLEDGMENT}

This research was conducted within the Max Planck School Matter to Life supported by the German Federal Ministry of Education and Research (BMBF) in collaboration with the Max Planck Society. J.B. is grateful for funding the European Research Council (ERC starting grant 852187). H.D. is grateful for funding from the European Research Council (ERC consolidator grant 724261). This project has received funding from the European Union's Horizon 2020 research and innovation programme under the Marie Skłodowska-Curie grant agreement No 765703 (to H.D. and A.M.). J.B. is grateful for an extensive discussion with R. Dean Astumian (Univ. Maine)

\section{ABBREVIATIONS}

EDC: 1-ethyl-3-(3-dimethylaminopropyl) carbodiimide; EDU: 1-(3(dimethylamino)propyl)-3-ethylurea; Ac-D-OH: N-acetyl Aspartic Acid

\section{REFERENCES}

1. Rothemund, P. W. K., Folding DNA to create nanoscale shapes and patterns. Nature 2006, 440 (7082), 297-302.

2. Douglas, S. M.; Dietz, H.; Liedl, T.; Högberg, B.; Graf, F.; Shih, W. M., Self-assembly of DNA into nanoscale three-dimensional shapes. Nature 2009, 459 (7245), 414-418.

3. Dietz, H.; Douglas, S. M.; Shih, W. M., Folding DNA into Twisted and Curved Nanoscale Shapes. Science 2009, 325 (5941), 725.

4. Hong, F.; Zhang, F.; Liu, Y.; Yan, H., DNA Origami: Scaffolds for Creating Higher Order Structures. Chemical Reviews 2017, 117 (20), 12584 12640 .

5. Dong, J.; Zhou, C.; Wang, Q., Towards Active Self-Assembly Through DNA Nanotechnology. Top Curr Chem (Cham) 2020, 378 (2), 33.

6. Teller, C.; Willner, I., Functional nucleic acid nanostructures and DNA machines. Current Opinion in Biotechnology 2010, 21 (4), 376-391.

$7 . \quad$ Ramezani, H.; Dietz, H., Building machines with DNA molecules. Nature Reviews Genetics 2020, 21 (1), 5-26.

8. Gerling, T.; Wagenbauer, K. F.; Neuner, A. M.; Dietz, H., Dynamic DNA devices and assemblies formed by shape-complementary, non-base pairing 3D components. Science 2015, 347 (6229), 1446.

9. Zhang, D. Y.; Winfree, E., Control of DNA Strand Displacement Kinetics Using Toehold Exchange. Journal of the American Chemical Society 2009, 131 (47), 17303-17314.

10. Simmel, F. C.; Yurke, B.; Singh, H. R., Principles and Applications of Nucleic Acid Strand Displacement Reactions. Chem. Rev. (Washington, DC, U. S.) 2019, 119 (10), 6326-6369.

11. Li, J.; Johnson-Buck, A.; Yang, Y. R.; Shih, W. M.; Yan, H.; Walter, N. G., Exploring the speed limit of toehold exchange with a cartwheeling DNA acrobat. Nature Nanotechnology 2018, 13 (8), 723-729.

12. Zhang, D. Y.; Seelig, G., Dynamic DNA nanotechnology using strand-displacement reactions. Nature Chemistry 201 1, 3 (2), 103-113.

13. Del Grosso, E.; Ragazzon, G.; Prins, L. J.; Ricci, F., Fuel-Responsive Allosteric DNA-Based Aptamers for the Transient Release of ATP and Cocaine. Angew Chem Int Ed Engl 2019, 58 (17), 5582-5586.

14. Gentile, S.; Del Grosso, E.; Prins, L. J.; Ricci, F., Reorganization of Self-Assembled DNA-Based Polymers using Orthogonally Addressable Building Blocks*. Angew Chem Int Ed Engl 2021, 60 (23), 12911-12917.

15. Groeer, S.; Walther, A., Switchable supracolloidal 3D DNA origami nanotubes mediated through fuel/antifuel reactions. Nanoscale 2020, 12 (32), 16995-17004.

16. Kuzyk, A.; Yang, Y.; Duan, X.; Stoll, S.; Govorov, A. O.; Sugiyama, H.; Endo, M.; Liu, N., A light-driven three-dimensional plasmonic nanosystem that translates molecular motion into reversible chiroptical function. Nature Communications 2016, 7 (1), 10591.

17. Del Grosso, E.; Ponzo, I.; Ragazzon, G.; Prins, L. J.; Ricci, F., Disulfide-Linked Allosteric Modulators for Multi-cycle Kinetic Control of DNABased Nanodevices. Angew Chem Int Ed Engl 2020, 59 (47), 21058-21063.
18. Del Grosso, E.; Prins, L. J.; Ricci, F., Transient DNA-Based Nanostructures Controlled by Redox Inputs. Angew Chem Int Ed Engl 2020, 59 (32), 13238-13245.

19. Goepfrich, K.; Urban, M. J.; Frey, C.; Platzman, I.; Spatz, J. P.; Liu, N., Dynamic Actuation of DNA-Assembled Plasmonic Nanostructures in Microfluidic Cell-Sized Compartments. Nano Lett. 2020, 20 (3), 1571-1577.

20. Heinen, L.; Walther, A., Temporal control of i-motif switch lifetimes for autonomous operation of transient DNA nanostructures. Chem. Sci. 2017, 8 (5), 4100-4107.

21. Mariottini, D.; Del Giudice, D.; Ercolani, G.; Di Stefano, S.; Ricci, F., Dissipative operation of $\mathrm{pH}$-responsive DNA-based nanodevices. Chemical Science 2021.

22. Marras, A. E.; Shi, Z.; Lindell, M. G.; Patton, R. A.; Huang, C.-M.; Zhou, L.; Su, H.-J.; Arya, G.; Castro, C. E., Cation-Activated Avidity for Rapid Reconfiguration of DNA Nanodevices. ACS Nano 2018, 12 (9), 9484-9494.

23. Lauback, S.; Mattioli, K. R.; Marras, A. E.; Armstrong, M.; Rudibaugh, T. P.; Sooryakumar, R.; Castro, C. E., Real-time magnetic actuation of DNA nanodevices via modular integration with stiff micrH-levers. Nat. Commun. 2018, 9 (1), 1-11.

24. Kopperger, E.; List, J.; Madhira, S.; Rothfischer, F.; Lamb, D. C.; Simmel, F. C., A self-assembled nanoscale robotic arm controlled by electric fields. Science (Washington, DC, U. S.) 2018, 359 (6373), 296-301.

25. Ranallo, S.; Sorrentino, D.; Ricci, F., Orthogonal regulation of DNA nanostructure self-assembly and disassembly using antibodies. Nat Commun 2019, 10 (1), 5509 .

26. Green, L. N.; Subramanian, H. K. K.; Mardanlou, V.; Kim, J.; Hariadi, R. F.; Franco, E., Autonomous dynamic control of DNA nanostructure self-assembly. Nat Chem 2019, 11 (6), 510-520.

27. Yehl, K.; Mugler, A.; Vivek, S.; Liu, Y.; Zhang, Y.; Fan, M.; Weeks, E. R.; Salaita, K., High-speed DNA-based rolling motors powered by RNase H. Nature Nanotechnology 2016, 11 (2), 184-190.

28. Deng, J.; Walther, A., Fuel-Driven Transient DNA Strand Displacement Circuitry with Self-Resetting Function. Journal of the American Chemical Society 2020, 142 (50), 21102-21109.

29. Deng, J.; Walther, A., Pathway Complexity in Fuel-Driven DNA Nanostructures with Autonomous Reconfiguration of Multiple Dynamic Steady States. Journal of the American Chemical Society 2020, 142 (2), 685-689.

30. Feng, Y.; Ovalle, M.; Seale, J. S. W.; Lee, C. K.; Kim, D. J.; Astumian, R. D.; Stoddart, J. F., Molecular Pumps and Motors. Journal of the American Chemical Society 2021, 143 (15), 5569-5591.

31. Boekhoven, J.; Hendriksen, W. E.; Koper, G. J.; Eelkema, R.; van Esch, J. H., Transient assembly of active materials fueled by a chemical reaction. Science 2015, 349 (6252), 1075-9.

32. Tena-Solsona, M.; Riess, B.; Grotsch, R. K.; Lohrer, F. C.; Wanzke, C.; Kasdorf, B.; Bausch, A. R.; Muller-Buschbaum, P.; Lieleg, O.; Boekhoven, J., Non-equilibrium dissipative supramolecular materials with a tunable lifetime. Nat Commun 2017, 8, 15895.

33. Dai, K.; Fores, J. R.; Wanzke, C.; Winkeljann, B.; Bergmann, A. M.; Lieleg, O.; Boekhoven, J., Regulating Chemically Fueled Peptide Assemblies by Molecular Design. Journal of the American Chemical Society 2020, 142 (33), 14142-14149.

34. Kriebisch, B. A. K.; Jussupow, A.; Bergmann, A. M.; Kohler, F.; Dietz, H.; Kaila, V. R. I.; Boekhoven, J., Reciprocal Coupling in Chemically Fueled Assembly: A Reaction Cycle Regulates Self-Assembly and Vice Versa. $J$ Am Chem Soc 2020, 142 (49), 20837-20844.

35. Spath, F.; Donau, C.; Bergmann, A. M.; Kranzlein, M.; Synatschke, C. V.; Rieger, B.; Boekhoven, J., Molecular Design of Chemically Fueled PeptidePolyelectrolyte Coacervate-Based Assemblies. J Am Chem Soc 2021, 143 (12), 4782-4789.

36. Wanzke, C.; Jussupow, A.; Kohler, F.; Dietz, H.; Kaila, V. R. I.; Boekhoven, J., Dynamic Vesicles Formed By Dissipative Self-Assembly. ChemSystemsChem 2020, 2 (1), e1900044.

37. Borsley, S.; Leigh, D. A.; Roberts, B. M. W., A Doubly KineticallyGated Information Ratchet Autonomously Driven by Carbodiimide Hydration. Journal of the American Chemical Society 2021, 143 (11), 4414-4420.

38. Kariyawasam, L. S.; Hartley, C. S., Dissipative Assembly of Aqueous Carboxylic Acid Anhydrides Fueled by Carbodiimides. Journal of the American Chemical Society 2017, 139 (34), 11949-11955.

39. Schnitter, F.; Bergmann, A. M.; Winkeljann, B.; Rodon Fores, J.; Lieleg, O.; Boekhoven, J., Synthesis and characterization of chemically fueled supramolecular materials driven by carbodiimide-based fuels. Nature Protocols 2021. 
40. Tena-Solsona, M.; Janssen, J.; Wanzke, C.; Schnitter, F.; Park, H.; Rieß, B.; Gibbs, J. M.; Weber, C. A.; Boekhoven, J., Accelerated Ripening in Chemically Fueled Emulsions**. ChemSystemsChem 2021, 3 (2), e2000034.

41. Schnitter, F.; Boekhoven, J., A Method to Quench CarbodiimideFueled Self-Assembly. ChemSystemsChem 2021, 3 (1), e2000037.

42. Tsukanov, R.; Tomov, T. E.; Berger, Y.; Liber, M.; Nir, E., Conformational Dynamics of DNA Hairpins at Millisecond Resolution Obtained from Analysis of Single-Molecule FRET Histograms. The Journal of Physical Chemistry B 2013, 117 (50), 16105-16109.

43. Wallace, M. I.; Ying, L.; Balasubramanian, S.; Klenerman, D., NonArrhenius kinetics for the loop closure of a DNA hairpin. Proceedings of the National Academy of Sciences 2001, 98 (10), 5584-5589.

44. Grunwell, J. R.; Glass, J. L.; Lacoste, T. D.; Deniz, A. A.; Chemla, D. S.; Schultz, P. G., Monitoring the Conformational Fluctuations of DNA Hairpins Using Single-Pair Fluorescence Resonance Energy Transfer. Journal of the American Chemical Society 2001, 123 (18), 4295-4303.

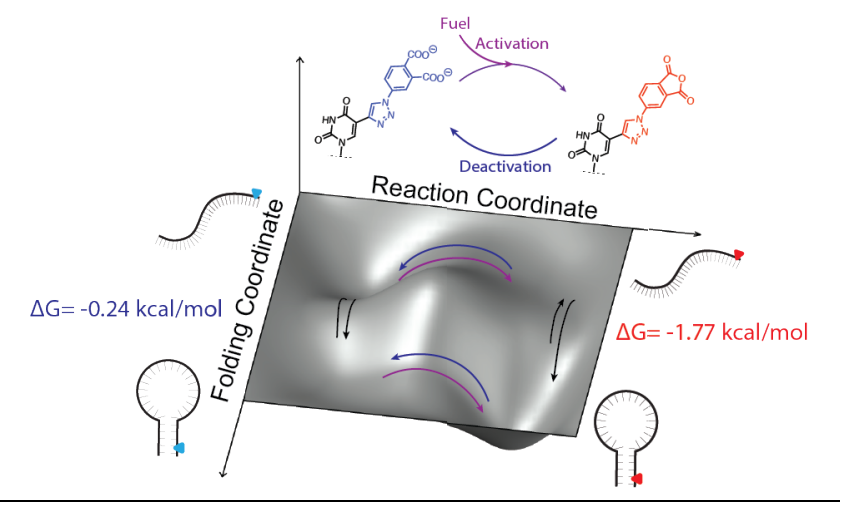

